\title{
Existence of multisite intrinsic localized modes in one-dimensional Debye crystals
}

\author{
V. Koukouloyannis ${ }^{1, *}$ and I. Kourakis ${ }^{2, \dagger}$ \\ ${ }^{1}$ School of Physics, Theoretical Mechanics, Aristotle University of Thessaloniki, 54124 Thessaloniki, Greece \\ ${ }^{2}$ Institut für Theoretische Physik IV, Fakultät für Physik und Astronomie, Ruhr Universität Bochum, D-44780 Bochum, Germany
}

(Received 15 January 2007; published 9 July 2007)

\begin{abstract}
The existence of highly localized multisite oscillatory structures (discrete multibreathers) in a nonlinear Klein-Gordon chain which is characterized by an inverse dispersion law is proven and their linear stability is investigated. The results are applied in the description of vertical (transverse, off-plane) dust grain motion in dusty plasma crystals, by taking into account the lattice discreteness and the sheath electric and/or magnetic field nonlinearity. Explicit values from experimental plasma discharge experiments are considered. The possibility for the occurrence of multibreathers associated with vertical charged dust grain motion in strongly coupled dusty plasmas (dust crystals) is thus established. From a fundamental point of view, this study aims at providing a rigorous investigation of the existence of intrinsic localized modes in Debye crystals and/or dusty plasma crystals and, in fact, suggesting those lattices as model systems for the study of fundamental crystal properties.
\end{abstract}

DOI: 10.1103/PhysRevE.76.016402 PACS number(s): 52.27.Lw, 63.20.Pw, 63.20.Ry, 05.45.-a

\section{INTRODUCTION}

Periodic lattices of interacting particles are known from solid state physics to sustain, apart from propagating vibrations (phonons), a variety of localized excitations, due to a mutual balance between the intrinsic nonlinearity of the medium and mode dispersion. Such structures, "traditionally" sought for and investigated in a continuum approximation (i.e., assuming that the typical spatial variation scale far exceeds the typical lattice scale, e.g., the lattice constant $r_{0}$ ), include nontopological solitons (pulses), kinks (i.e., shocks or dislocations), and localized modulated envelope structures (envelope solitons). Various generic nonlinear theories have been developed in order to investigate their occurrence in different physical contexts [1]. In addition to these (continuum) theories, which neglect discreteness for the sake of analytical tractability, attention has been paid since more than a decade ago to highly localized vibrating structures [discrete breathers (DBs) or intrinsic localized modes (ILMs)], which owe their existence to the lattice discreteness. Following some pioneering ILM related works in the late 1980's (see, e.g., in Refs. [2-6]), the breakthrough in the theoretical study of DBs took place with the appearance of the first rigorous proofs of discrete breather existence, furnished independently by MacKay and Aubry [7] (who introduced the notion of continuation from a suitable anticontinuous limit) and Flach [8] (using a homoclinic orbit approach). The former method was later extended later in Ref. [9]. The discrete vibrational state in which the energy of a localized excitation is concentrated in more than one sites is called a multibreather. An extensive study on the existence and stability of multibreathers can be found in Refs. [10-14], while

\footnotetext{
*Electronic address: vkouk@physics.auth.gr; http://users.auth.gr/ $\sim$ vkouk

${ }^{\dagger}$ Research partly carried out at: Universiteit Gent, Sterrenkundig Observatorium, Krijgslaan 281, B-9000 Gent, Belgium; Electronic address: ioannis@tp4.rub.de; www.tp4.rub.de/ ioannis
}

as regards the homoclinic method approach on the same subject one is referred to Refs. $[15,16]$. A large number of noteworthy detailed studies have appeared, elucidating various aspects involved in the spontaneous formation, mobility and interaction of DBs, both theoretically and experimentally; see, e.g., Refs. [17-21] for a review.

Recent studies of collective processes in a dustcontaminated plasma (DP) [22-24] have revealed a variety of new linear and nonlinear collective effects, which are observed in laboratory and space dusty plasmas. An issue of particular importance in DP research is the formation of strongly coupled DP crystals by highly charged dust grains, typically in the sheath region above a horizontal negatively biased electrode in experiments $[22,25]$. Low-frequency oscillations are known to occur in gas-discharge experiments [25] involving these mesoscopic dust grain quasilattices, in the longitudinal (in-plane, acoustic mode), horizontal transverse (in-plane), and vertical transverse (off-plane) directions [22-25]. Here, we shall only focus on the latter (transverse) degree of freedom, which was first predicted in Refs. [26,27] (also see Ref. [28]) and later experimentally observed.

Nonlinearity is an intrinsic feature of dust crystal dynamics. It may be due either to inter-grain (Debye-type, screened electrostatic) nonlinear interactions, or to geometric effects, i.e., to coupling among different modes [29]. Finally, and rather importantly, nonlinearity is also due to the plasma sheath environment, which provides the electric [25] and/or magnetic [30] forces ensuring equilibrium by suspending the crystal at a levitated position above the negative electrode; thus, the electric/magnetic forces in the sheath provide a "substrate" potential which is intrinsically anharmonic (see discussion below). From the point of view of nonlinear science, therefore, DP crystal dynamics provide a blend of anharmonic on-site potential and coupling nonlinearity, accounting for a unique hybrid among nonlinear Klein-Gordon type and, say, Fermi-Pasta-Ulam (FPU) type theories (see the discussion in Ref. [31]). Despite this fact, present day knowledge of nonlinear mechanisms related to dust lattice modes is admittedly still in a preliminary stage. Small amplitude localized longitudinal excitations (described by a Boussinesq 
equation for the longitudinal grain displacement $u$ or a Korteweg-de Vries equation for the density $\partial u / \partial x$ ) were considered in Refs. [32] and generalized in Ref. [33]. Also, the amplitude modulation of longitudinal $[34,35]$ and transverse (vertical, off-plane) $[36,37]$ dust lattice waves (LDLW, TDLW, respectively) was recently considered. A model for nonlinear DP lattice dynamics taking into account transverseto-longitudinal mode coupling was suggested in Ref. [29]. Those studies were recently extended to diatomic-like Debye (bi)crystals [38] and eventually extended to more realistic, hexagonal crystalline geometries [39]. All of these studies have relied on a quasicontinuum description of dust lattice dynamics. The discrete character of dust-lattice oscillations has, to our best knowledge, not yet been studied, apart from a recent first investigation which was restricted to singlemode transverse dust breathers [40]. That study has examined the properties of vertical (off-plane, transverse) dust lattice vibrations, from first principles. A description of such a system, using the homoclinic method approach, was formulated in Ref. [41], providing some results on breathers in Debye crystals. The existence of breathers associated to longitudinal (in-plane, acoustic) charged particle motion in a Debye crystal was discussed in Ref. [31], although a detailed investigation from first principles (which would be the ana$\log$ of the Fermi-Pasta-Ulam problem, tailor-fit to Debye or dust crystals) is still missing.

Most interestingly, the transverse (linear) dust lattice mode is known to obey an inverse dispersion law: therefore the group velocity $v_{g}=\omega^{\prime}(k)$ and the phase velocity $v_{p h}$ $=\omega / \mathrm{k}$ point towards opposite directions. The anharmonic character of the vertical on-site potential (confirmed experimentally $[42,43]$ ), in combination with the high discreteness of dust crystals, clearly suggested by experiments $[44,45]$, may play an important role in mechanisms such as energy localization, information storage, and response to external excitations. Although all of the ingredients prescribing the occurrence of discrete breathers in DP crystals seem to be present, rather surprisingly, this aspect of DP dynamics still remains essentially unexplored.

From a fundamental point of view, crystalline arrangements of charged particles (Debye crystals and/or dusty plasma lattices) provide an excellent toy model for the study of nonlinear excitations in atomicscale lattices, or in ionic scale crystalline structures [e.g., ultracold plasmas (UCPs)], since they mimic atomic chain dynamics at a scale which is appropriate for human observation (dust crystals can even be filmed and analyzed via simple and inexpensive video image processing; see, e.g., in Ref. [25]). Here, we aim to stress that, as regards Discrete Breather excitations, such lattices may provide a convenient mesoscopic analogue of atomic chains, which should allow for direct observation of localized excitations and eventually in situ confirmation of analytical theories, via purpose-built experiments. To our best knowledge, no other mesoscopic or macroscopic system permitting such experimental modeling freedom exists.

In this study, we are interested in investigating the conditions for the occurrence of discrete multisite lattice excitations (multibreathers) in a nonlinear (infinite sized) KleinGordon-like chain, which is characterized by an inverse dispersion law. Nonlinearity is supplied by a (nonharmonic) on-site potential, while interparticle interactions are taken to be linear. A negative coupling coefficient ("spring constant") value is assumed, in account of an inverse dispersion. Our results are applied in a description of real transverse dustlattice excitations, as observed in plasma discharge experiments. The formalism, the analytic tools and the numerical methods used in the present study are described in Refs. $[11,13,14]$ and allow us to evaluate the range of values of relevant physical parameters which may account for breather formation.

\section{EXISTENCE AND STABILITY OF MULTISITE INTRINSIC LOCALIZED MODES}

Our aim is to prove the existence of multibreather excitations in Debye crystals. The method we adopt is based on the continuation of a specific state of a suitable anticontinuous limit, as, e.g., in Refs. [7,9]. The formalism used is described in Ref. [11]. A brief outline of the method is provided in the following.

Consider the Hamiltonian describing a chain consisting of infinitely many identical nonlinear oscillators, with displacement $x_{i}$ and on-site potential $V\left(x_{i}\right)$, possessing a stable equilibrium at $x_{i}=0$, i.e., $V_{i}^{\prime}(0)=0$ and $V_{i}^{\prime \prime}{ }_{i}(0)=\omega_{p}^{2}>0$ (unit mass is assumed in the notation), coupled through a coupling constant $\epsilon$

$$
H=H_{0}+\epsilon H_{1}=\sum_{i=-\infty}^{\infty}\left(\frac{1}{2} p_{i}^{2}+V\left(x_{i}\right)\right)+\frac{\epsilon}{2} \sum_{i=-\infty}^{\infty}\left(x_{i+1}-x_{i}\right)^{2} .
$$

The corresponding equations of motion are

$$
\ddot{x}_{i}=-V^{\prime}\left(x_{i}\right)+\epsilon\left(x_{i+1}-2 x_{i}+x_{i-1}\right) \quad \forall i \in \mathbb{Z} .
$$

This is the classical Klein-Gordon chain, which is well known to support multibreather solutions. Note that, the multibreather existence theorems, based on a continuation from a suitable anticontinuous limit $[10,11]$ hold for an $\epsilon$ neighborhood around zero, and are thus valid either for $\epsilon$ $>0$ or for $\epsilon<0$, provided that $|\epsilon| \ll 1$.

Consider the integrable anticontinuous limit $(\epsilon=0)$, i.e., the chain is consisting of uncoupled oscillators. In this limit we consider the state where all the oscillators lie in equilibrium apart from $n+1$ "central" ones which lie on periodic orbits satisfying the resonance condition

$$
\frac{\omega_{0}}{k_{0}}=\cdots=\frac{\omega_{n}}{k_{n}}=\omega, \quad k_{i} \in \mathbb{Z} .
$$

This state is time periodic with period $T=2 \pi / \omega$ and trivially space localized. We seek the conditions under which this state will be continued for $\epsilon \neq 0$ by keeping the previously mentioned attributes, providing this way a multibreather. At this limit, the motion of the central oscillators is described by

$$
\begin{aligned}
w_{i} & =\omega_{i} t+\vartheta_{i}, \\
J_{i} & =\text { const }, \quad i=0, \ldots, n,
\end{aligned}
$$

where $\left(w_{i}, J_{i}\right)$ are the action angle-variables of the uncoupled oscillators, $\vartheta_{i}$ are the initial angles, and $\omega_{i}$ are the corre- 
sponding angular frequencies. The $T$-periodic motion, which is described by Eq. (3), can be continued for $\epsilon \neq 0$ small enough, to form a $T$-periodic $(n+1)$-site breather, provided that the following conditions hold.

(1) The anharmonicity condition of the individual oscillators, i.e., $d \omega_{i} / d J_{i} \neq 0$, at least in the neighborhood of the specific periodic orbit.

(2) The nonresonance condition $\omega_{p} \neq m \omega, \forall m \in \mathbb{N}$, where $\omega_{p}$ denotes the linear frequency of the single oscillator. However, even if both of these conditions hold, not all the states of the anticontinuous limit will be continued to a multibreather. In addition, the phases of the oscillators in this limit must be such that the system of equations

$$
\frac{\partial\left\langle H_{1}\right\rangle}{\partial \phi_{i}}=0, \quad i=1, \ldots, n
$$

has simple zeros, i.e., it is also required that $\operatorname{det}\left|\partial^{2}\left\langle H_{1}\right\rangle / \partial \phi_{i} \partial \phi_{j}\right| \neq 0$, where $\phi_{i}=k_{i} \vartheta_{i-1}-k_{i-1} \vartheta_{i}$ is a generalization of the notion of phase difference between the successive oscillators, in order to include resonances other that the 1:1. Here,

$$
\left\langle H_{1}\right\rangle=\int_{0}^{T} H_{1} d t
$$

is the average value of the perturbative term of the Hamiltonian calculated along a periodic orbit of the uncoupled system over a time period.

Since we consider identical oscillators, as it is thoroughly explained in Ref. [14], Eq. (4) can be written as

$$
\frac{\partial\left\langle H_{1}\right\rangle}{\partial \phi_{i}}=0 \Leftrightarrow \sum_{m=1}^{\infty} m A_{k_{i} m} A_{k_{i-1} m} \sin m \phi_{i}=0,
$$

where $A_{j}$ is the $j$ th Fourier coefficient of the single oscillator. From Eq. (6), we conclude that $\phi_{i}=0$ and $\phi_{i}=\pi$ always satisfy Eq. (4) while, if special symmetry conditions hold, one could also obtain additional solutions which would be time nonreversible, following the terminology of Ref. [7].

If the action-angle canonical transformation is known, one could search for these solutions in Eq. (4) or its equivalent (6). However, in the generic case where the explicit form of the action-angle variables is not known, a method to calculate the necessary quantities has been developed in Ref. [13]. According to this method, the system of Eq. (4) is equivalent to the following one:

$$
\int_{0}^{T} \frac{\partial H_{1}}{\partial x_{i}} p_{i} d t=0, \quad i=1, \ldots, n .
$$

This system can easily be solved numerically, as will be later shown in a specific example.

In addition to the existence of the multibreather solutions, the phase difference between the oscillators also determines its linear stability, as shown in Refs. [12,14]. The linear stability of a periodic orbit (which in the specific case is the anticipated multibreather), is defined by the eigenvalues of the corresponding Floquet matrix $\lambda_{i}$ (called also Floquet multipliers), see, e.g., Ref. [17]. If all these eigenvalues lie on the unit circle of the complex plane then the periodic orbit is linearly stable, while if an eigenvalue lies outside the circle the orbit is unstable. Note that for every eigenvalue we also have its reciprocal and its complex conjugate, because of the Hamiltonian structure of the system (i.e., for every $\lambda_{i}$, there are also $\lambda_{i}^{-1}, \lambda_{i}^{*}$, and $\lambda_{i}^{*-1}$ ). So, we cannot have just one eigenvalue outside the unit circle but only an even number of them. For $\epsilon=0$, the abovementioned eigenvalues lie in two complex conjugate bundles at $e^{ \pm i \omega_{p} T_{b}}$, except the $2 n+2$ eigenvalues which correspond to the $n+1$ central oscillators which lie at unity. For $|\epsilon| \neq 0 \ll 1$, the eigenvalues of the noncentral oscillators move along the unit circle being of the same Krein kind, forming this way the phonon band, while the ones of the central oscillators are given by

$$
\lambda_{i}=e^{\sigma_{i} T},
$$

where $\sigma_{i}$ are the corresponding $2 n+2$ characteristic exponents. As it was proven in Ref. [10] (and also stated, within the present formalism, in Ref. [11]), these exponents are given in the leading order of approximation by

$$
\sigma_{i}^{2}=\epsilon \sigma_{i 1}^{2}+O\left(\epsilon^{3 / 2}\right),
$$

where $\sigma_{i 1}^{2}$ coincide with the $n+1$ eigenvalues of the stability matrix

$$
E=-A \cdot B,
$$

with

$$
A=\left(\frac{\partial^{2}\left\langle H_{1}\right\rangle}{\partial \vartheta_{i} \partial \vartheta_{j}}\right), \quad B=\left(\frac{\partial^{2} H_{0}}{\partial J_{i} \partial J_{j}}\right) .
$$

Therefore, if the various values of $\sigma_{i 1}^{2}$, i.e., the eigenvalues of $E$, are distinct, and the product $\epsilon \sigma_{i 1}^{2}$ is negative, then the corresponding characteristic exponents $\sigma_{i}$ are imaginary up to leading order terms and distinct, which results in eigenvalues $\lambda_{i}$ on the unit circle and the multibreather is linearly stable. If there are no other solutions than the standard ones the corresponding linear stability is well defined by the knowledge of the resonant angles $\phi_{i}$, the kind of potential anharmonicity-i.e., hardening $\left(\partial \omega_{i} / \partial J_{i}>0\right)$ or softening $\left(\partial \omega_{i} / \partial J_{i}<0\right)$-and the sign of $\epsilon$. Let us now apply this method in a specific example, namely, the equation of transverse dust grain motion in a dust crystal.

\section{TRANSVERSE PARTICLE MOTION IN DEBYE CRYSTALS AND DUSTY PLASMA CRYSTALS}

We shall consider the vertical (off-plane, $\sim \hat{z}$ ) charged particle displacement in a crystal (assumed quasi-onedimensional, of infinite length: identical grains of charge $q$ and mass $M$ are situated at $x_{i}=i r_{0}$, where $i=\ldots,-2$, $-1,0,1,2, \ldots$.$) , by taking into account the intrinsic nonlin-$ earity of the sheath electric (and/or magnetic) potential. The in-plane (longitudinal acoustic $\sim \hat{x}$ and shear $\sim \hat{y}$ ) degrees of freedom are assumed suppressed; this situation is indeed today realized in appropriate experiments $[44,45]$, where a laser impulse triggers transverse dust grain oscillations, while a confinement potential ensures the chain's in-plane stability. 


\section{A. Equation of motion}

The vertical grain displacement obeys an equation in the form $[22,36,37,40]$

$$
\begin{aligned}
\frac{d^{2} \delta z_{n}}{d t^{2}} & +\nu \frac{d z_{n}}{d t}+\omega_{0}^{2}\left(\delta z_{n+1}+\delta z_{n-1}-2 \delta z_{n}\right)+\omega_{g}^{2} \delta z_{n}+\alpha\left(\delta z_{n}\right)^{2} \\
& +\beta\left(\delta z_{n}\right)^{3}=0
\end{aligned}
$$

where $\delta z_{n}(t)=z_{n}(t)-z_{0}$ denotes the small displacement of the $n$th grain around the (levitated) equilibrium position $z_{0}$, in the transverse $(z)$ direction. The characteristic frequency $\omega_{0}$ $=\left[-q \Phi^{\prime}\left(r_{0}\right) /\left(M r_{0}\right)\right]^{1 / 2}$ results from the dust grain (electrostatic) interaction potential $\Phi(r)$, e.g., for a Debye-Hückel potential $[46,47] \quad \Phi_{D}(r)=(q / r) e^{-r / \lambda_{D}}$, one has $\omega_{0, D}^{2}$ $=q^{2} /\left(M r_{0}^{3}\right)\left(1+r_{0} / \lambda_{D}\right) \exp \left(-r_{0} / \lambda_{D}\right)$, where $\lambda_{D}$ denotes the effective DP Debye radius [22]. The damping coefficient $\nu$ accounts for dissipation due to collisions between dust grains and neutral atoms. The gap frequency $\omega_{g}$ and the nonlinearity coefficients $\alpha, \beta$ are defined via the overall vertical force $F(z)=F_{e / m}-M g \approx-M\left[\omega_{g}^{2} \delta z_{n}+\alpha\left(\delta z_{n}\right)^{2}+\beta\left(\delta z_{n}\right)^{3}\right]+O\left[\left(\delta z_{n}\right)^{4}\right]$, which has been expanded around $z_{0}$ by formally taking into account the (anharmonicity of the) local form of the sheath electric (follow exactly the definitions in Ref. [36], not reproduced here) and/or magnetic [48] field(s), as well as, possibly, grain charge variation due to charging processes [37]. Recall that the electric and magnetic levitating force(s) $F_{e / m}$ balance(s) gravity at $z_{0}$. Notice the difference in structure from the usual nonlinear Klein-Gordon equation used to describe one-dimensional oscillator chains—see, e.g., Eq. (1) in Ref. [5]: TDLWs (phonons) in this chain are stable only in the presence of the field force $F_{e / m}$.

For convenience, the time and vertical displacement variables may be scaled over appropriate quantities, i.e., the characteristic (single grain) oscillation period $\omega_{g}^{-1}$ and the lattice constant $r_{0}$, respectively, viz. $t=\omega_{g}^{-1} \tau$ and $\delta z_{n}=r_{0} q_{n}$; Eq. (10) is thus expressed as

$$
\frac{d^{2} q_{n}}{d \tau^{2}}+\epsilon\left(q_{n+1}+q_{n-1}-2 q_{n}\right)+q_{n}+\alpha^{\prime} q_{n}^{2}+\beta^{\prime} q_{n}^{3}=0
$$

where the (dimensionless) damping term, now expressed as $\left(\nu / \omega_{g}\right) d q_{n} / d \tau \equiv \nu^{\prime} \dot{q}_{n}$, will be henceforth omitted in the lefthand side (assuming $\nu^{\prime} \ll 1$ ). The coupling parameter is now $\epsilon=\omega_{0}^{2} / \omega_{g}^{2}$, and the nonlinearity coefficients are now: $\alpha^{\prime}$ $=\alpha r_{0} / \omega_{g}^{2}$ and $\beta^{\prime}=\beta r_{0}^{2} / \omega_{g}^{2}$. All quantities in Eq. (11) are dimensionless [50].

\section{B. Linear transverse dust-lattice waves}

Retaining only the linear contribution and considering oscillations of the type, $\delta z_{n} \sim \exp \left[i\left(k n r_{0}-\omega t\right)\right]+$ c.c. (complex conjugate) in Eq. (10), one obtains the well known transverse dust lattice (TDL) wave optical-mode-like dispersion relation

$$
\omega^{2}=\omega_{g}^{2}-4 \omega_{0}^{2} \sin ^{2}\left(\frac{k r_{0}}{2}\right)
$$

or

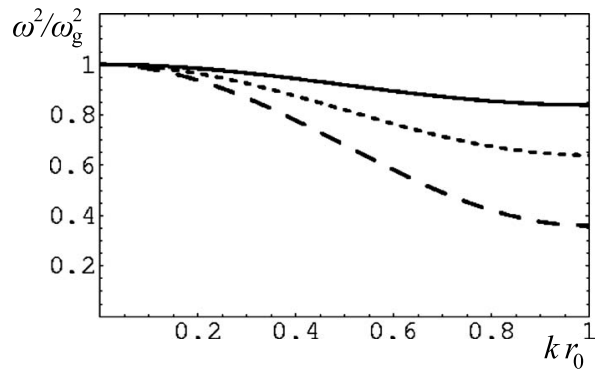

FIG. 1. The dispersion relation of the TDL excitations: frequency square (normalized by $\omega_{g}^{2}$ ), as given by (12), vs (reduced) wave number $k r_{0}$. The value of $\omega_{0} / \omega_{g}(\sim$ coupling strength $)$ increases from top to bottom. Note that upper (less steep, continuous) curve is more likely to occur in a real (weakly coupled) DP crystal.

$$
\widetilde{\omega}^{2}=1-4 \epsilon \sin ^{2}(\tilde{k} / 2) .
$$

See that the wave frequency $\omega \equiv \tilde{\omega} \omega_{g}$ decreases with increasing wave number $k=2 \pi / \lambda \equiv \tilde{k} / r_{0}$ (or decreasing wavelength $\lambda$ ), see Fig. 1, implying that transverse vibrations propagate as a backward wave: the group velocity $v_{g}$ $=\omega^{\prime}(k)$ and the phase velocity $\omega_{\mathrm{ph}}=\omega / k$ have opposite directions (this behavior has been observed in recent experiments). The modulational stability profile of these linear waves (depending on the plasma parameters) was investigated in Refs. [36,37]. Notice the natural gap frequency $\omega(k=0)=\omega_{g}=\omega_{\max }$, corresponding to an overall motion of the chain's center of mass, as well as the cutoff frequency $\omega_{\min }=\left(\omega_{g}^{2}-4 \omega_{0}^{2}\right)^{1 / 2} \equiv \omega_{g}\left(1-4 \epsilon^{2}\right)^{1 / 2}$ (obtained at the end of the first Brillouin zone $k=\pi / r_{0}$ [49]) which is absent in the continuum limit, viz. $\omega^{2} \approx \omega_{g}^{2}-\omega_{0}^{2} k^{2} r_{0}^{2}$ (for $k \ll r_{0}^{-1}$ ); obviously, the study of wave propagation in this $\left(k \lesssim \pi / r_{0}\right)$ region invalidates the continuum treatment employed so far in literature. The essential feature of discrete dynamics, to be retained here, is the (narrow) bounded TDLW (phonon) frequency band, limited in the interval $\omega \in\left[\left(\omega_{g}^{2}-4 \omega_{0}^{2}\right)^{1 / 2}, \omega_{g}\right]$; note that one thus naturally obtains the stability constraint: $\omega_{0}^{2} / \omega_{g}^{2}=\epsilon<1 / 4$ (so that $\omega \in \mathbb{R} \forall k \in\left[0, \pi / r_{0}\right]$ ).

We need not go into further details concerning the linear regime, since it is covered rather extensively in the literature [22]. We shall, instead, see what happens if the nonlinear terms are retained, in this discrete description.

\section{Multibreathers in Debye crystals and Dusty plasma crystals}

Equation (11) can be generated by a Hamiltonian of the form (1) by considering a quartic on-site (sheath) polynomial potential of the form

TABLE I. Experimental data: Sets I, II, and III of sheath potential anharmonicity values, as obtained from the data in Ref. [43]. Column IV provides the data in Ref. [42].

\begin{tabular}{ccccc}
\hline \hline & & Ref. [43] & & Ref. [42] \\
\hline & I & II & III & IV \\
$\alpha^{\prime}$ & 0.02 & 0.016 & -0.27 & -0.5 \\
$\beta^{\prime}$ & -0.16 & -0.17 & -0.03 & 0.07 \\
\hline \hline
\end{tabular}






(a)
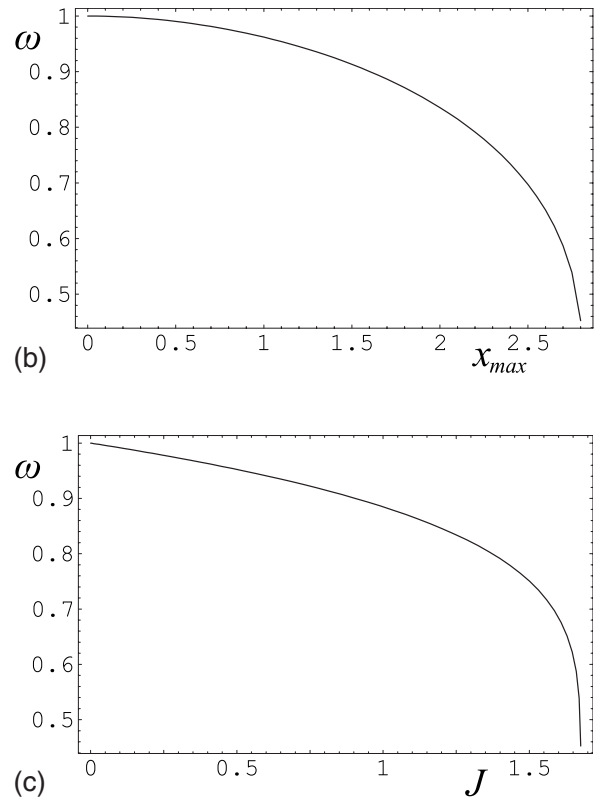

FIG. 2. (a) The on-site potential is depicted against the vertical displacement $x$. (b) The frequency $\omega$ is depicted against the amplitude of the oscillation $x_{\max }$. (c) The frequency $\omega$ is depicted against the action variable $J$. All the figures have been obtained by using the set of values III in Table I.

$$
V\left(x_{i}\right)=\frac{1}{2} x_{i}^{2}+\frac{\alpha^{\prime}}{3} x_{i}^{3}+\frac{\beta^{\prime}}{4} x_{i}^{4},
$$

and considering negative values of $\epsilon$ (in account of inverse dispersion). Note that, henceforth $x$ stands for $q$ of Eq. (11), i.e., describes the normalized vertical displacement from the equilibrium.

The values of the anharmonicity parameters $\alpha^{\prime}$ and $\beta^{\prime}$ may be deduced from dusty plasma experiments on nonlinear vertical dust lattice oscillations [42-45]. For instance, the Kiel (Germany) experiment by Zafiu et al. [43]—using a laser to trigger nonlinear vertical dust grain oscillations-has provided the values $\alpha / \omega_{g}^{2}=+0.02 ;+0.016 ;-0.27\left(\mathrm{~mm}^{-1}\right)$ and $\beta / \omega_{g}^{2}=-0.16 ;-0.17 ;-0.03\left(\mathrm{~mm}^{-2}\right)$ (successively, by gradually increasing the diameter of the dust grains; see Table 1 in Ref. [43]). In our notation, this implies $\alpha^{\prime}$ $\simeq+0.02 ;+0.016 ;-0.27$, and $\beta^{\prime} \simeq-0.16 ;-0.17 ;-0.03$ (for a lattice spacing of the order of $r_{0} \simeq 1 \mathrm{~mm}$ ). Note that damping was very low $\left(\nu^{\prime} \simeq 0.02\right)$, thus a posteriori justifying our neglecting it.
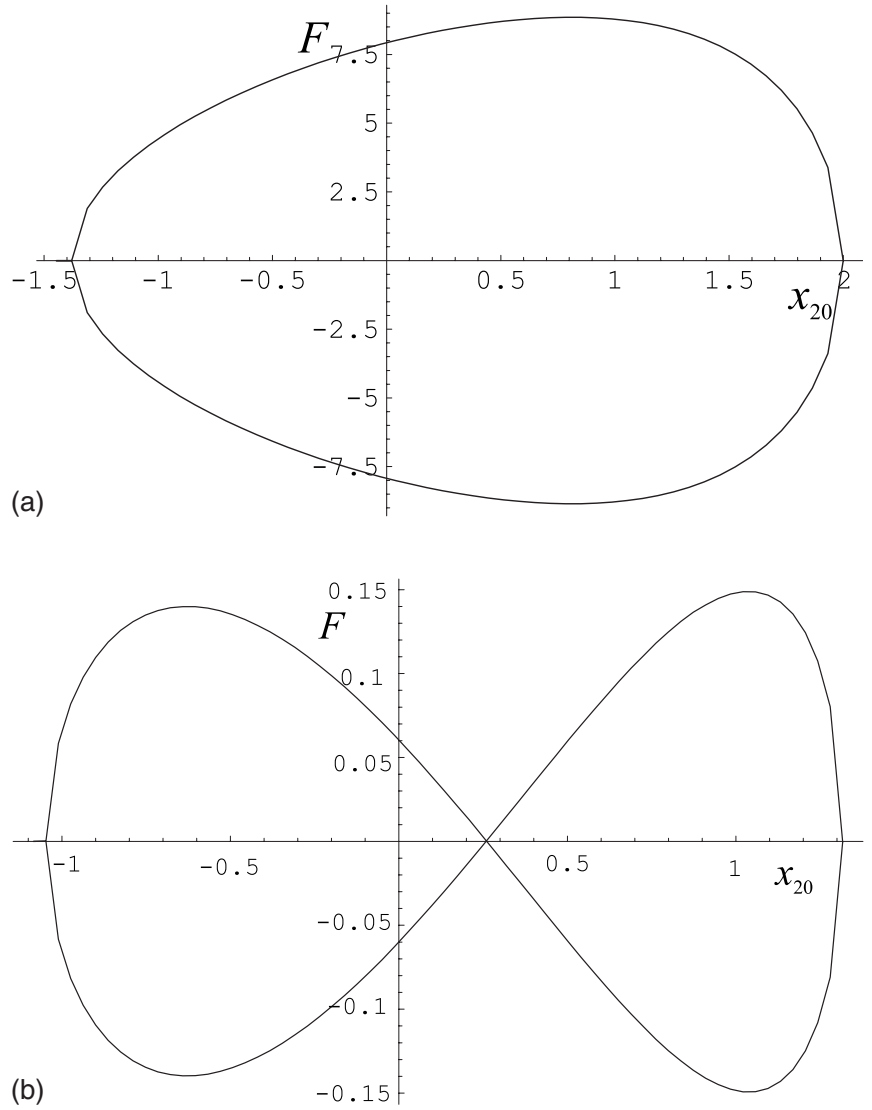

FIG. 3. $F\left(x_{20}\right)$ for the $1: 1$ and 2:3 resonances.

The experiment on anharmonic single grain oscillations by Ivlev et al. [42], carried out in Garching (Germany), provides curve fitting data for $\Phi(z)$, i.e., $\alpha / \omega_{g}^{2}=-0.5 \mathrm{~mm}^{-1}$ and $\beta / \omega_{g}^{2}=0.07 \mathrm{~mm}^{-2}$. One thus deduces $\alpha^{\prime} \approx-0.5$ and $\beta^{\prime}$ $\approx 0.07$ (for a lattice spacing, say typically, of the order of $\left.r_{0} \approx 1 \mathrm{~mm}\right)$. Note that the damping coefficient $\nu$ was as low as $\nu / 2 \pi \simeq 0.067 \mathrm{~s}^{-1}$, so that (with $\omega_{g} / 2 \pi \simeq 17 \mathrm{~s}^{-1}$ ) one has $\nu^{\prime}=\nu / \omega_{g} \simeq 0.004$ (the pressure in that experiment was kept as low as $0.5 \mathrm{~Pa}$; see the original paper for technical details on the experimental device). The sets of anharmonicity values are shown together in Table I, for reference.

The results of the experiment on linear TDLWs by Misawa et al. [44] allows for a rough estimation of the coupling strength (still for low pressure; see details in Ref. [44]): $\omega_{g} \simeq 155 \mathrm{~s}^{-1}$ and $\omega_{0} \simeq 19.5 \mathrm{~s}^{-1}$ [derived from Fig. 3(a) therein], which give $\epsilon \simeq 0.016$. Note that the effective damping term was kept as low as $\nu \simeq 0.239 \mathrm{~s}^{-1}$, i.e., $\nu^{\prime}=\nu / \omega_{g}$ $\simeq 0.00154$.

We will first examine the system provided by Zafiu et al. in Ref. [43]. Since the first three sets of values presented in Table I provide similar results we shall consider only the results in the third column, i.e., $\alpha^{\prime}=-0.27$ and $\beta^{\prime}=-0.03$. The on-site potential $V(x)$ as a function of the vertical displacement $x$ is depicted in Fig. 2(a). The frequency $\omega$ dependence on the maximum displacement of the free oscillator $x_{\max }$ is shown in Fig. 2(b), while its dependence on the action $J$ is shown in Fig. 2(c). Note that, since there is a one to one correspondence between $x_{\max }$ and J, Figs. 2(b) and 2(b) are equivalent. Due to the form of the potential $V(x)$, the region 

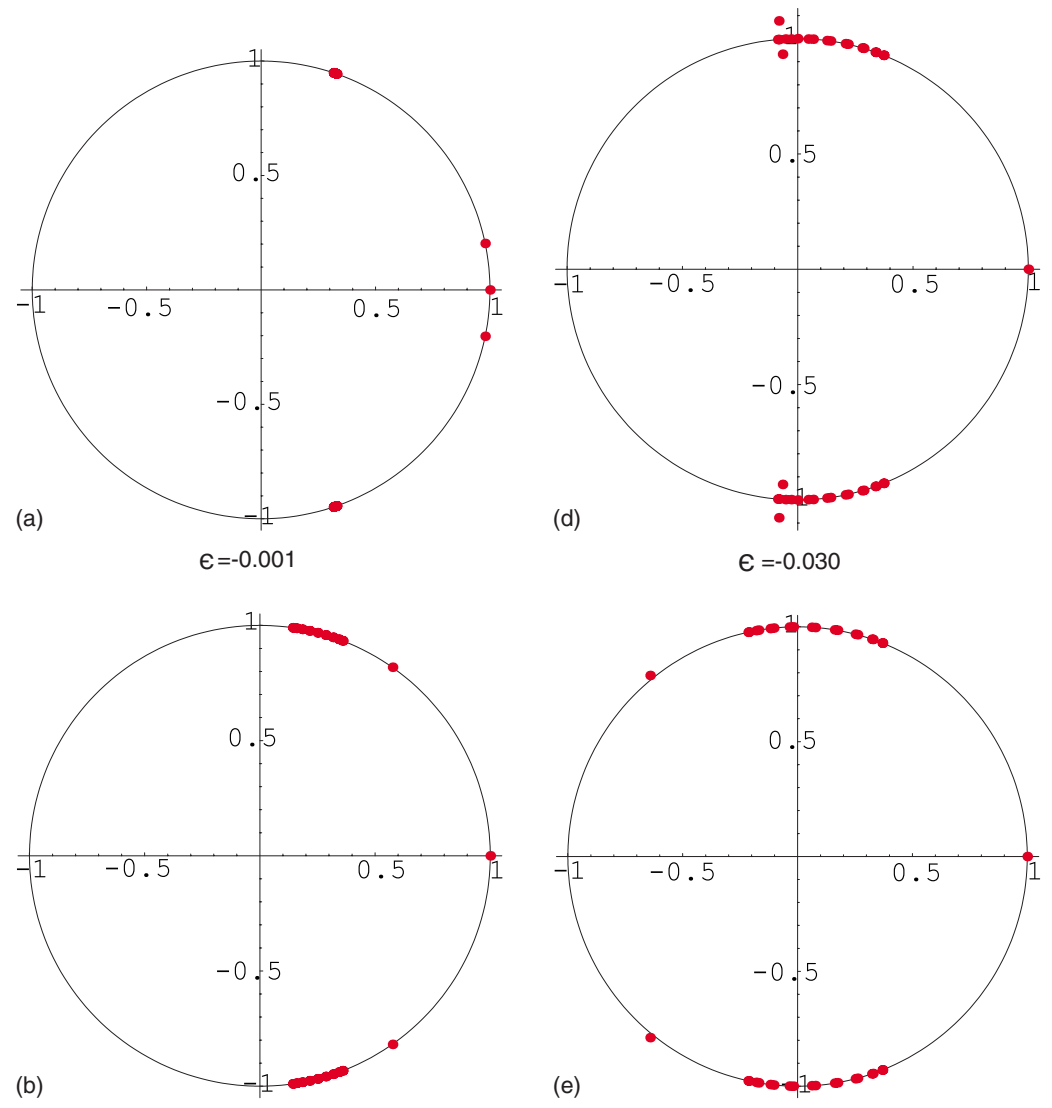

FIG. 4. (Color online) The destabilization scenario represented by the eigenvalues of the Floquet matrix for growing values of $|\epsilon|$.

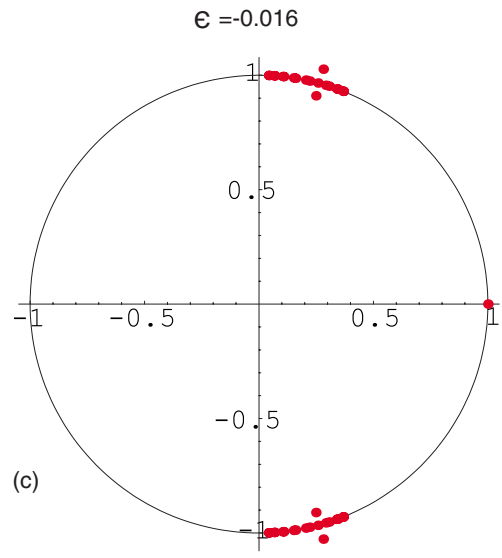

$\epsilon=-0.022$

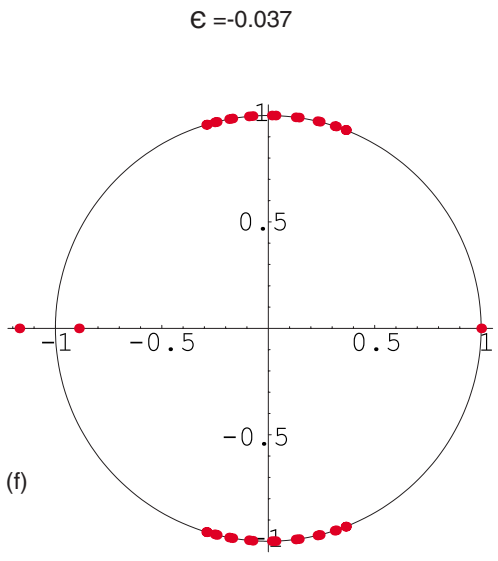

$\epsilon=-0.041$ of motion is restricted to the region from $x \simeq-1.55$ to $x$ $\simeq 2$.82. Let us remark, in passing, that this potential form essentially accounts for crystal dissociation (melting), which occurs when vibrating particles overcome the finite potential barrier. We note that $\omega(J)$ is a decreasing function of $J$, so the anharmonicity condition $\partial \omega / \partial J \neq 0$ is satisfied everywhere in the allowed region of motion. The computations of $\omega(x)$ and $\omega(J)$ has been made numerically since the explicit action-angle transformation is not known; for a more detailed description, see Ref. [13]. In Fig. 2, $\omega(J)$ together with the potential function of the specific choice of coefficients are shown. We consider the anticontinuous limit of only two oscillators moving in resonant periodic orbits with frequencies $\omega_{1}$ and $\omega_{2}$ and the rest in the equilibrium. We examine the 1:1 and 2:3 cases, i.e., the cases with $\omega=\omega_{1}=\omega_{2}$ and $\omega$ $=\frac{\omega_{1}}{2}=\frac{\omega_{2}}{3}$. We are thus simply left with the task of choosing appropriate values of the amplitude $x_{\max }$, corresponding to the two values of the frequency $\omega_{1}$ and $\omega_{2}$, which should fulfil the resonance condition and, at the same time, the frequency $\omega$ should not be close to the harmonic vibration frequency $\omega_{p}$ (here 1, i.e., the linear frequency in the absence of coupling). This is easily accomplished upon simple inspection of Fig. 2(b) or 2(c). Note that we have to avoid a neighborhood of values around $\omega_{p}$, since for $\epsilon \neq 0$ a phonon band will be formed instead of the single frequency $\omega_{p}$ and possible resonance of the breather frequency with this band will end up in energy loss via the excitation of linear waves, so that localized discrete modes will be destabilized. By fixing 


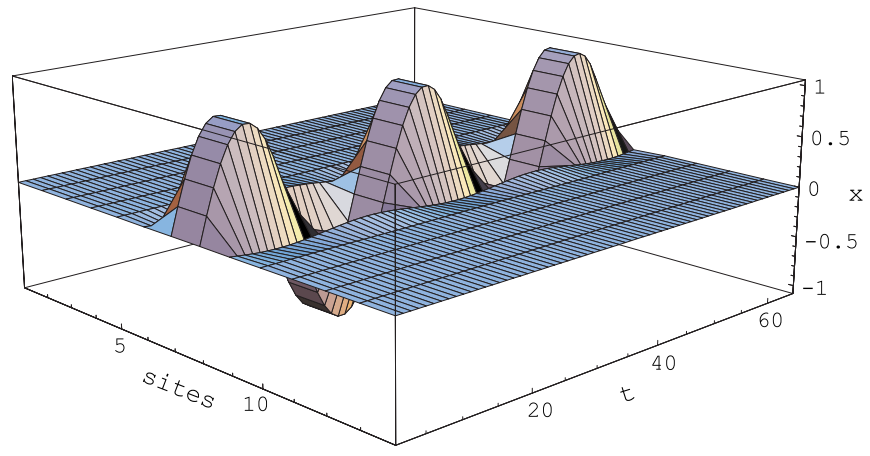

FIG. 5. (Color online) Time evolution of a 1:1 two-breather for $\epsilon=-0.016$.

the orbits of the two oscillators we determine at the same time the energy of the orbits at the anticontinuous limit as well as the period $T$ of the produced multibreather. We now have to check which phase difference the central oscillators must have in order to form a multibreather for $\epsilon \neq 0$.

We now have to check condition (4). Since we consider two oscillators in the anticontinuous limit there is only one angle $\phi$ and, as already shown above, the $\phi=0, \pi$ solutions always exist. Since the action-angle transformation is not analytically known, in order to check for extra solutions, one has to solve the equivalent equation (7), i.e., for $i=2$,

$$
\int_{0}^{T} \frac{\partial H_{1}\left(x_{1}, x_{2}\right)}{\partial x_{2}} p_{2} d t=0 .
$$

Note that all of the above calculations are made in the anticontinuous limit $(\epsilon=0)$. Since the periodic orbits are given, the above equation defines a relationship between the initial conditions of the two orbits $x_{10}, p_{10}, x_{20}, p_{20}$. We fix $x_{10}=0$ and choose the value of $p_{10}>0$ which is specified by the periodic orbit. So, the only free variable is $x_{20}$, since $p_{20}$ can be accordingly calculated from the equation of energy. Equation (15) now leads to [13]

$$
F\left(x_{20}\right)=\int_{0}^{T} x_{1} p_{2} d t=0 .
$$

This equation is two branched, i.e., yields one branch for each choice of sign for the momentum $p_{20}$. In Fig. 3 , these two branches are presented together in the same diagram for the $1: 1$ as well as for the $2: 3$ resonance.

For the $1: 1$ resonance we see that $F\left(x_{20}\right)$ has two roots, which correspond to the standard breather solutions $\phi=0, \pi$. As for the stability of these solutions, following the arguments in Refs. [12,14], the solution with $z=0$ will be the linearly stable one among these two and, since there are no other solutions besides the ones already mentioned, this will be the only linearly stable solution. In particular, in Ref. [14] it is shown that $\sigma_{11}$ in Eq. (9) is given by

$$
\sigma_{11}^{2}=-\frac{\partial \omega}{\partial J} \sum_{m=1}^{\infty} m^{2} A_{m}^{2} \cos m \phi,
$$

which for $\phi=0, \frac{\partial \omega}{\partial J}<0$ and $\epsilon<0$ provides imaginary characteristic exponents $\pm \sigma_{1}$ up to $O(\sqrt{\epsilon})$ and consequently linearly

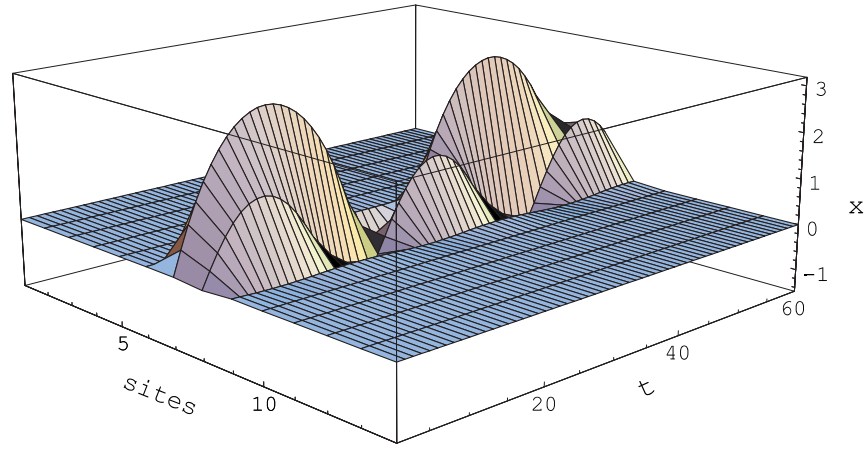

FIG. 6. (Color online) Time evolution of a 2:3 two-breather for $\epsilon=-0.003$.

stable multibreather, which confirms what has been claimed above. Note that Eq. (17) [in combination with Eq. (9)] is essentially equivalent (for $\phi=0$ and 1:1 resonance) to the simple stability criterion

$$
\epsilon \frac{\partial \omega}{\partial J}>0
$$

We have computed this solution for only two central oscillators, but the procedure would be exactly analogous for any number $n$ of central oscillators since, as shown in Ref. [13], the system consists of independent equations. In that case, the only linearly stable solution would be $\phi_{i}=0$, for $i$ $=1, \ldots, n$.

The abovementioned solution is proven to be linearly stable for values of the coupling parameter $\epsilon$ close enough to zero. However, as the absolute value of $\epsilon$ increases, the central oscillators eigenvalues move along the unit circle, while the noncentral oscillators eigenvalues will also move along the opposite direction, forming this way the phonon band. As the value of $|\epsilon|$ is increasing, at some point the central oscillator eigenvalue will penetrate the phonon band and, since they are of opposite Krein sign, they can leave the unit circle forming a complex quadruple (see, e.g., Ref. [17]); the multibreather thus becomes unstable, since there will be four eigenvalues outside the unit circle. By further increasing $|\epsilon|$ the eigenvalues will return to the unit circle restabilizing the multibreather, which remains stable until the eigenvalues collide at -1 and leave the unit circle along the real axis causing a period doubling bifurcation and consequently, the final destruction of the breather. This destabilization scenario is shown in Fig. 4.

Anticipating an experimental confirmation of our results, the experimentally measured value of the coupling constant $\epsilon$ should be inside the stability regions. By choosing the amplitude of the oscillation at the anticontinuous limit to be $x_{\max } \simeq 1.51$, a stable multibreather is achieved for $\epsilon=0.016$, as it can be seen in Fig. 4; this fact confirms that this kind of motion can be supported by the specific model. The time evolution of the resulting two-breather is depicted in Fig. 5.

Let us examine the 2:3 resonance now. Note that, in Fig. 3 the curve $F\left(x_{20}\right)$ crosses the $x$ axis four times. These four roots correspond to only two values of $\phi$ only, namely, the trivial ones $\phi=0$ and $\pi$. The resulting multibreathers, which 



FIG. 7. (a) The on-site potential is depicted against the vertical displacement $x$. (b) The frequency $\omega$ is depicted against the amplitude of the oscillation $x_{\max }$. (c) The frequency $\omega$ is depicted against the action variable $J$. All the figures have been obtained using the set of values IV in Table I.

have the form of Fig. 6, are very quickly (for small values of $\epsilon)$ destabilized so they are physically meaningless and will not be examined any further.

Consider now the nonlinearity values given by Ivlev et al. in Ref. [42]. The corresponding potential and frequency functions are shown in Fig. 7. In this case, the frequency $\omega(J)$ is not everywhere a monotonous function of $J$ but presents a minimum at $J \simeq 2.3$. If we constrain the allowed region of motion up to the minimum of $\omega$, then we obtain similar results to the abovementioned. So, we can obtain stable 1:1 symmetric multibreathers. However, if we extend the allowed region of motion, we can consider a 1:1 anticontinuous limit with the oscillators moving with different amplitudes but the same frequency. This limit is continued for $\epsilon \neq 0$ to provide a nonsymmetric 1:1 2-breather as it is shown in Fig. 8.

\section{CONCLUSIONS}

This study was devoted to an investigation, from first principles, of the existence of discrete multisite lattice exci-
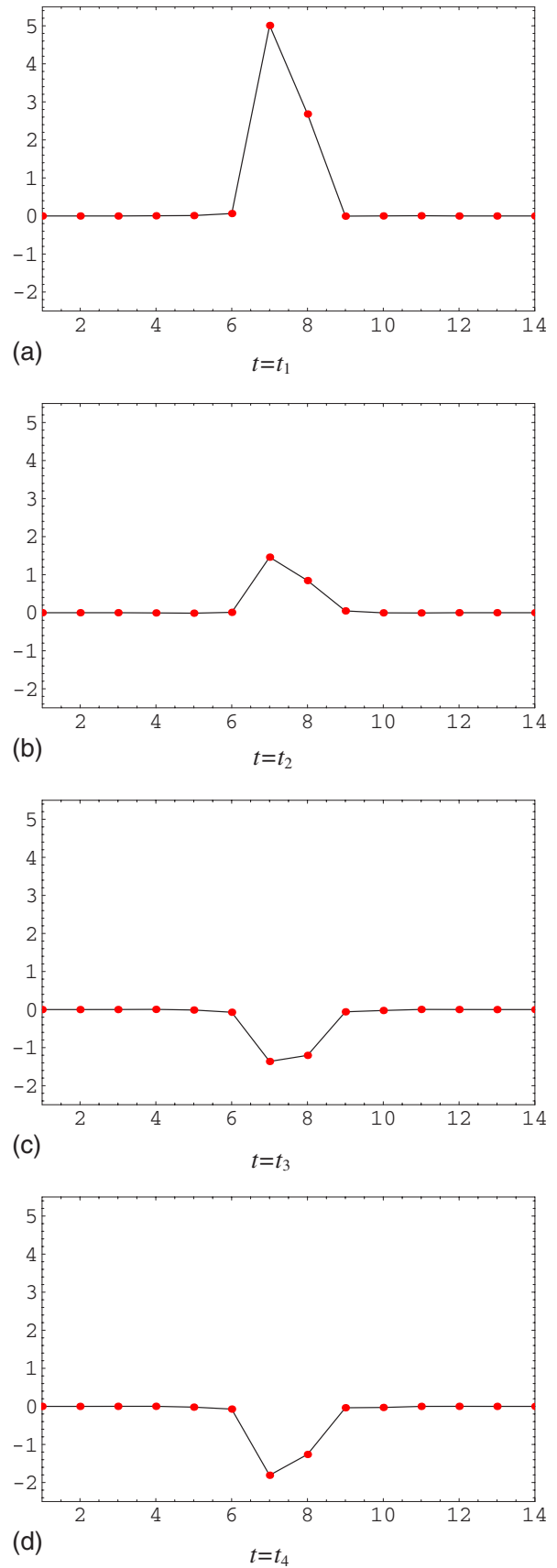

FIG. 8. (Color online) Snapshots of an nonsymmetric multibreather for four successive time moments.

tations (multibreathers) in a Debye (e.g., dusty plasma) crystal. Relying on the analytical and numerical methodology established in Refs. $[11,13,14]$, we have shown that Debye crystals may, indeed, support multisite highly localized oscillatory motion (multibreathers). In particular, employing nonlinearity parameters provided by plasma discharge experiments $[42,43]$, we have established that $1: 1$ symmetric and nonsymmetric multibreathers should be expected, while multibreathers having other resonances are expected to be unstable and thus physically meaningless.

The methodology which was used to extract these results is quite general and is directly applicable to a wide variety of systems. It was shown that the relative strength of electro- 
static interparticle coupling as compared to the substrate potential harmonicity, here expressed by the parameter $\epsilon$, may play a (de)stabilizing role vis-a-vis discrete breather excitations. In discharge experiments, the parameter $\epsilon$ may be tuned by adapting the coupling (e.g., via the grain surface potential, which determines the charge state $Z$ ) and the sheath electrostatic potential (by modifying plasma parameters such as density or pressure). Therefore, the results of our study may straightforward be tested (and will hopefully be confirmed) by appropriate experiments.

From a purely fundamental point of view, one of the principal aims of this work was to stress the fact that Debye lattices (today realized as dust-lattices in simple experimental devices) provide a flexible test field, available at a convenient mesoscopic or macroscopic level, for various theoretical and computational predictions now available concerning oscillatory motion in discrete systems.

Further extensions of our study should take into account dissipation (here neglected) and/or higher dimensionality effects (e.g., triangular two-dimensional configurations, as ob- served in experiments [25]). Studies in this direction are under way, and will be reported soon.

\section{ACKNOWLEDGMENTS}

This work was supported by the research program PYTHAGORAS II of the Greek Ministry of Education and the E.U. One of us (I.K.) acknowledges funding from the FWO (Fonds Wetenschappelijk Onderzoek-Vlaanderen, Flemish Research Fund) during a visiting appointment at the Sterrenkundig Observatorium, University of Gent (Belgium). $\mathrm{He}$ also acknowledges support from the Deutsche Forschungsgemeinschaft (DFG, Germany) under the EmmyNoether program (Grant No. SH 93/3-1), during the latter stages of this work. Finally, the authors would like to thank the Max-Planck institute for the Physics of Complex Systems in Dresden and especially S. Flach for accommodating the last part of this work during the "Nonlinear Physics in Periodic Structures and Metamaterials" conference.
[1] T. Dauxois and M. Peyrard, Physics of Solitons (Cambridge University Press, Cambridge, 2005).

[2] S. Takeno, K. Kisoda, and A. J. Sievers, Prog. Theor. Phys. Suppl. 94, 242 (1988).

[3] J. B. Page, Phys. Rev. B 41, 7835 (1990).

[4] T. Dauxois and M. Peyrard, Phys. Rev. Lett. 70, 3935 (1993).

[5] Yu. Kivshar, Phys. Lett. A 173, 172 (1993).

[6] D. K. Campbell and M. Peyrard, CHAOS_Soviet American Perspectives on Nonlinear Science, edited by D. K. Campbell (AIP, Physics New York, 1990).

[7] R. S. MacKay and S. Aubry, Nonlinearity 7, 1623 (1994).

[8] S. Flach, Phys. Rev. E 51, 1503 (1995).

[9] J.-A. Sepulchre and R. S. MacKay, Nonlinearity 10, 679 (1997).

[10] T. Ahn, R. S. MacKay, and J.-A. Sepulchre, Nonlinear Dyn. 25, 157 (2001)

[11] V. Koukouloyannis and S. Ichtiaroglou, Phys. Rev. E 66, 066602 (2002).

[12] J. F. R. Archilla, J. Cuevasa, B. Sanchez-Rey, and A. Alvarez, Physica D 180, 235 (2003).

[13] V. Koukouloyannis, Phys. Rev. E 69, 046613 (2004).

[14] V. Koukouloyannis and S. Ichtiaroglou, Int. J. Bifurcation Chaos Appl. Sci. Eng. 16, 1823 (2006).

[15] T. Bountis, H. W. Capel, M. Kollmann, J. C. Ross, J. M. Bergamin, and J. P. van der Weele, Phys. Lett. A 268, 50 (2000).

[16] P. Panagopoulos, T. Bountis, and C. Skokos, J. Vibr. Acoust. 126, 520 (2004).

[17] S. Aubry, Physica D 103, 201 (1997).

[18] S. Flach and C. R. Willis, Phys. Rep. 295, 181 (1998).

[19] R. S. MacKay, Physica A 288, 174 (2000).

[20] See various articles in the Volume (Focus Issue): Chaos 13, 586 (2003).

[21] D. K. Campbell, S. Flach, and Yu. S. Kivshar, Phys. Today 57, (2004).

[22] P. K. Shukla and A. A. Mamun, Introduction to Dusty Plasma
Physics (Institute of Physics, Bristol, 2002).

[23] S. V. Vladimirov and K. Ostrikov, Phys. Rep. 393, 175 (2004).

[24] S. V. Vladimirov, K. Ostrikov, and A. A. Samarian, Physics and Applications of Complex Plasmas (Imperial College Press, London, 2005).

[25] G. E. Morfill, H. M. Thomas, and M. Zuzic, in Advances in Dusty Plasma Physics, edited by P. K. Shukla, D. A. Mendis, and T. Desai (World Scientific, Singapore, 1997), p. 99.

[26] S. V. Vladimirov, P. V. Shevchenko, and N. F. Cramer, Phys. Rev. E 56, R74 (1997).

[27] S. V. Vladimirov, P. V. Shevchenko, and N. F. Cramer, Phys. Plasmas 5, 4 (1998).

[28] S. V. Vladimirov, N. F. Cramer, and P. V. Shevchenko, Phys. Rev. E 60, 7369 (1999).

[29] I. Kourakis and P. K. Shukla, Phys. Scr. 113, 97 (2004).

[30] S. V. Vladimirov, G. E. Morfill, V. V. Yaroshenko, and N. F. Cramer, Phys. Plasmas 10, 2659 (2003).

[31] I. Kourakis and P. K. Shukla, Int. J. Bifurcation Chaos Appl. Sci. Eng. 16, 1711 (2006).

[32] F. Melandsø, Phys. Plasmas 3, 3890 (1996).

[33] I. Kourakis and P. K. Shukla, Eur. Phys. J. D 29, 247 (2004).

[34] M. R. Amin, G. E. Morfill, and P. K. Shukla, Phys. Plasmas 5, 2578 (1998); Phys. Scr. 58, 628 (1998).

[35] I. Kourakis and P. K. Shukla, Phys. Plasmas 11, 1384 (2004).

[36] I. Kourakis and P. K. Shukla, Phys. Plasmas 11, 2322 (2004).

[37] I. Kourakis and P. K. Shukla, Phys. Plasmas 11, 3665 (2004).

[38] I. Kourakis, P. K. Shukla, and G. E. Morfill, Phys. Plasmas 12, 112104 (2005).

[39] B. Farokhi, I. Kourakis, and P. K. Shukla, Phys. Plasmas 13, 122304 (2006).

[40] I. Kourakis and P. K. Shukla, Phys. Plasmas 12, 014502 (2005).

[41] I. Kourakis, P. K. Shukla, and V. Basios (unpublished), http:// hal.ccsd.cnrs.fr/ccsd-00001892/en/

[42] A. V. Ivlev, R. Sütterlin, V. Steinberg, M. Zuzic, and G. Mor- 
fill, Phys. Rev. Lett. 85, 4060 (2000).

[43] C. Zafiu, A. Melzer, and A. Piel, Phys. Rev. E 63, 066403 (2001).

[44] T. Misawa, N. Ohno, K. Asano, M. Sawai, S. Takamura, and P. K. Kaw, Phys. Rev. Lett. 86, 1219 (2001).

[45] B. Liu, K. Avinash, and J. Goree, Phys. Rev. Lett. 91, 255003 (2003).

[46] U. Konopka, G. E. Morfill, and L. Ratke, Phys. Rev. Lett. 84, 891 (2000).

[47] E. B. Tomme, D. A. Law, B. M. Annaratone, and J. E. Allen,
Phys. Rev. Lett. 85, 2518 (2000).

[48] In the magnetically levitated dust crystal case, the reader should consider the definitions in Ref. [37], upon setting $K_{1}$ $\rightarrow \alpha, K_{2} \rightarrow \beta$ and $K_{3} \rightarrow 0$, therein.

[49] Charles Kittel, Introduction to Solid State Physics, 8th ed. (Wiley, New York, 2004).

[50] Note, for rigor, that the coupling strength $\epsilon$ in Eq. (11) is formally the opposite of $\epsilon$ in Eq. (2), hence the inversedispersive character discussed in the text. We shall clarify this subtlety in notation further below. 